Schriftenreihe herausgegeben vom

Forschungszentrum Populäre Musik

der Humboldt-Universität zu Berlin

in: PopScriptum 9 - Instrumentalisierungen - Medien und ihre Musik

\title{
Distanzierte Verhältnisse? Zur Musikinstrumentalisierung der Reproduktionsmedien
}

\author{
Rolf Großmann, Deutschland
}

\author{
"An einer systematischen Ordnung der Musikinstrumente sind in erster Linie \\ die Musikhistoriker, die Ethnologen und die Verwalter völkerkundlicher \\ und kulturhistorischer Sammlungen interessiert." [1]
}

In der Tat sind es die Professionals der Konservierung, für deren berufliche Tätigkeit systematische Ordnungen elementar sind. Weniger an Ordnung interessiert waren dagegen die Performer der jamaikanischen Soundsystems, die DJs der New Yorker Discos, die HipHopper der Bronx und mit ihnen die Jugendkultur des Pop, welche die E-Gitarren beiseite legte und das DJ-Set zum Symbol einer neuen musikalischen Praxis werden ließ. Dass es sich bei diesem party- und cluborientierten Sounderzeuger gar nicht um ein ordentliches Instrument handelte, sondern um ein Medium, das noch bis vor kurzem seine große Treue ('High Fidelity') zu den von ihm abgebildeten Originalen beteuerte, war nebensächlich. Dabei war die Entwicklung schon im Sprachgebrauch vorhersehbar: auf einer Party 'die Musik zu machen', meinte schon in den 1980ern keineswegs den Griff zum Instrument oder die Mitwirkung in einer Band, man war stattdessen mit 'Auflegen' an der Reihe. Ein deutlicher Hinweis darauf, dass auch die Reproduktionsmedien eine Aufführungspraxis begründen, also etwas, das dem instrumentalen Spiel vorbehalten schien.

\section{Geordnete Verhältnisse}

Während die Systematik der Instrumente mit den Elektrophonen, zu denen die E-Gitarren und die Synthesizer gezählt werden sollten, bereits ans Ende gekommen zu sein schien, ging der technikkulturelle Wandel musikalischer Praxis also unübersehbar weiter. Die Instrumentenkunde, für welche die Kategorisierung der physikalischen Entstehung der Klänge immer eine zentrale Rolle spielte, hatte den Idiophonen, den Membranophonen, den Chordophonen und den Aerophonen zuletzt eine Kategorie hinzu gefügt, die das in den ersten Dekaden des 20. Jahrhunderts immer bedeutender werdende physikalische Prinzip, die Elektrizität, ins System integrieren sollte. Mit den Elektrophonen sollten die Klänge und Instrumente der Elektrizität ihren Platz unter den vertrauten Klangerzeugern einnehmen. Dass sich hier eine Reihe systematischer Probleme ergeben konnte, war man bereits von den bestehenden Kategorien her gewohnt. Wenn also die elektrische Gitarre eher ein Chordophon sein könnte, das seine Schwingungen elektromechanisch über einer Magnetspule erzeugt, diese dann elektronisch verstärkt über einen Lautsprecher, also ein Membranophon, als 
Schwingungserzeuger der Schallwellen hörbar werden lässt, war das eine Problemkonstellation der Systematik, die von anderen Instrumenten bekannt war. So konnte man etwa bei der Klarinette darüber nachdenken, ob das Holzblatt nach Anregung selbst klingt, als Membran gespannt ist, oder ob erst die Luftsäule oder gar erst das Holz der gesamten Klarinette die Luft ursächlich in Schwingungen versetzt. Solche Fragen lassen sich natürlich ausführlich diskutieren, und wie jeder Archivar weiß, dass viele Gegenstände der einen Abteilung unter Umständen auch in der anderen Abteilung gut aufgehoben wären, so ist ebenso klar, dass die letztlich getroffene Zuordnung dennoch sinnvoll und richtig ist, wenn sie im Hinblick auf einen systematisch formulierten Sinnzusammenhang getroffen wurde. Auch ohne einen wissenschaftstheoretischen Exkurs zur 'Ord-nung der Dinge' ist es heute evident, dass Erkenntnisrahmen und Ordnungsprinzipien jeder Systematik korrespondieren. Dass die Rechnung einer Systematik unter dem Vorzeichen der Klangerzeugung jedoch nicht mehr aufgeht, wenn die Elektrizität ins Spiel kommt, hat eine einfache und nicht wegdiskutierbare Ursache: Sie erzeugt keine Klänge. Ihre Spannungsverläufe sind als solche nicht wahrnehmbar. Jede Anwendung von Elektrizität im Prozess der Klangerzeugung, sei sie elektromechanisch oder elektronisch, bedarf der Vermittlung in die Welt der Schallwellen. Physikalisch gesehen wird der Klang erst erzeugt, wenn ein durch die Elektrizität angeregter Gegen $\neg$ stand im Schallmedium Luft zu schwingen beginnt. Die Memb-ran eines Lautsprechers erfüllt allerdings genau dann ihren Zweck, wenn sie keinen Eigen-klang hat. Weder Elektrizität noch Lautsprecher geben daher an sich Hinweise auf einen spezifischen Klang, der ihnen den Platz in einer Systematik zuweisen könnte. Die Relevanz der Kategorie Klangerzeugung ergab sich allein durch die 'natürliche' physische Beschaffenheit eines Instruments und die Dominanz dieser physischen Grundlage für die weiteren Felder der Betrachtung, die möglichen Klangspektren, die Spielweise, die Eignung für die bereits entwickelten Kompositions- und Rezeptionsstrategien und viele andere. Mit der technischen Entwicklung der Mechanik verschiebt sich diese scheinbar naturgegebene Relation. Bereits bei Klappen, Ventilen, Klaviermechanik oder Orgelregistern ist zu beobachten, was elektrische Klangerzeugung und Sampling schließlich endgültig vollziehen: die Entkoppelung des physischen Spiels der Instrumente von der physischen Erzeugung der Klänge. Ein Drumcomputer und ein Percussioninstrument produzieren ähnliche Klänge, ihre Klangerzeugungsmechanismen und Spielweisen sind jedoch höchst unterschiedlich und im Falle des Computers von der physischen Erzeugung des Klangs vollständig entkoppelt. [2]

In dieser Situation sind die Erkenntnisfragen, die im vorliegenden Beitrag an eine spezifische Gruppe von Instrumenten des 20. und beginnenden 21. Jahrhunderts gestellt werden sollen, denn auch weniger museal und archivarisch als am Bedürfnis orientiert, die Veränderungen einer instrumentalen Praxis musikalischer Gestaltung besser $\mathrm{zu}$ verstehen, mit einem wissenschaftlich fundierten Verständnis $\mathrm{zu}$ begleiten und vielleicht erste systematische Überle-gungen anzustellen. Das Erkenntnisinteresse wird sich auf die Relation von Mensch, Instru-ment und musikalischer Kultur richten, in der physikalische Funktionsprinzipien zwar eine wichtige Rolle spielen, jedoch nicht dominant sind. Im Zentrum steht demgegenüber die Veränderung der Relation zwischen musikalischem Handeln, seinen (technischen) Werkzeugen und der erzeugten klingenden Struktur. Eine von den elektronischen Medien geprägte Lebenswelt transformiert verständlicherweise nicht nur die Bedingungen der Rezeption von Musik, sondern auch die Prozesse musikalischer Gestaltung in und mit diesen Medien. 


\section{Halbfertige Musik}

Zwei Aspekte dieses Wandels der instrumentalen Gestaltung von Musik sind hier von besonderer Bedeutung und ziehen ähnliche Konsequenzen in der Relation Mensch-Instrument nach sich:

- die Instrumentalisierung von Konfigurationen der Reproduktionsmedien sowie

- die Instrumentalisierung automatisch ablaufender (programmierter) algorithmischer Prozesse für die musikalische Gestaltung.

Beide Instrumentalisierungen (die anders gesagt eine 'Musikinstrumentwerdung' technischer Konfigurationen darstellen) stehen auf den ersten Blick quer zu unseren vertrauten Vorstellungen von einem Musikinstrument und das, obwohl E-Gitarre, Keyboard, DJ-Set und seit einiger Zeit Laptop gängige Elemente der Bühnenperformances sind. Während die E-Gitarre noch weit gehend mit ihrem nicht elektrischen Vorgänger-Instrument verwandt zu sein scheint und den konventionellen Erwartungen lediglich dort Probleme bereitet, wo das Feedback selbst als instrumental gestalteter Klang auftritt - etwa bei Jimi Hendrix oder dem Drone der Metal Machine Music Lou Reeds -, sind ihre genannten Nachfolger Boten einer völlig anderen Praxis musikalischer Gestaltung. Nur ein Aspekt zeigt sich bereits beim Feedback der E-Gitarre: Ihr Klang muss vom Spieler nicht selbst erzeugt, sondern aus der Rückkopplungsschleife heraus geformt werden, der Gestaltungsprozess wird eher zum Vorgang der Filterung, der Echtzeitselektion von Klängen aus sich ständig selbst erneuernden Oberton-kaskaden. Die mit DJ-Set, digitalen Keyboards und Computerinterfaces performant gestaltete musikalische Struktur ist dagegen nur noch zum Teil Ergebnis der Interaktion des Men-schen mit dem Instrument, sie ist zum anderen Teil bereits durch vorgängige gestalterische Arbeit vorformuliert, ihre Zeitgestalt nur begrenzt veränderbar. Der Zugriff auf eine halbfertige Musik, die dem Instrument bereits latent eingeschrieben ist, entbindet den Menschen der Notwendigkeit der physischen Ausführung aller Details der Klangformung, das Instrument schafft eine neue Distanz zwischen dem physischen Gestaltungsprozess und der physischen Gestalt der erklingenden Musik. Gleichzeitig, dies liegt in der Natur des Umgangs mit den komplexen Strukturen phonographisch gespeicherter oder algorithmisch generierter Musik, werden rationale Distanz und reflexive Durchdringung sehr bald integraler Teil des musikali-schen Spiels, da die gestalterischen Prozesse ein gewisses Maß an Planung notwendiger-weise vorauszusetzen scheinen.

"Der physische Kontakt des Menschen zur jeweiligen Form der Klangerzeugung und Klangformung verliert bei verschiedenen Instrumentengattungen zunehmend die ursprüngliche Unmittelbarkeit, die Abnahme der Körpernähe eines Instruments ist weitgehend kongruent zum Grad seiner konstruktiven Komplexität." [3]

Diese Abkoppelung der Klänge von der Physis und der direkten motorischen Aktion der Musizierenden widerspricht unserer Vorstellung eines Musikinstruments. Ab einer gewissen Stufe dieser Distanzierung scheint sich - wie Shintaro Miyazaki treffend beschreibt [4] - das Instrument in ein Medium zu verwandeln, welches eine präformierte Struktur lediglich vermittelt.

\section{Reproduktionsmusik - Musikautomat und Phonograph}

In dieser Situation liegt also die Frage nahe, ob und inwieweit dann überhaupt noch von Musikinstrumenten gesprochen werden kann. Neu ist diese Frage nicht, sie führt zurück in die 
Ära der Musikautomaten. Gerade zur Blütezeit der Spieldosen und Pianolas im 19. Jahrhundert gehen Musik und Technik unter dem Vorzeichen der romantischen Musikauffassung eine Hassliebe ein: technisch rationale Planung und Ausführung sollen vom solfeggio des Virtuosen bis zur Bühnentechnik des Musiktheaters reibungslos und hoch effizient verlaufen, jedoch keineswegs als solche hör- oder sichtbar werden. Gleichzeitig war die Entwicklung der Feinmechanik soweit fortgeschritten, dass komplexe musikalische Strukturen von $\mathrm{Mu}-$ sikmaschinen reproduziert werden konnten. Ernstzunehmende Aufführungen von Musikautomaten mit und ohne menschlichen Interpret wurden Teil musikalischer Kultur, die Relation von Musikinstrument und Maschine veränderte sich. Waren diese neuen Automaten auch Musikinstrumente?

Ein Grenzfall, der die Frage nach dem Instrumentcharakter der Musikautomaten zuspitzt, ist die Reduktion der Performanz auf das Betätigen des Einschalthebels. Bernd Enders - aus seinem Beitrag stammte auch das vorausgegangene Zitat - hat sich bereits Ende der 1980er Jahre, damals im Zuge der ideologischen Scharmützel der musikwissenschaftlichen Auseinandersetzung um die Berechtigung hochtechnisierter Musikinstrumente in der ernsten Musik, ausführlich mit dem Gedanken der Entfremdung und Entkörperlichung, einer möglichen Regression differenzierten instrumentalen Spiels im Zuge der Entwicklung elektronischer und programmierbare Instrumente auseinander gesetzt und auch diesen Fall berücksichtigt.

"Bei dieser Instrumentengruppe (der Musikautomaten, R.G.) beschränkt sich der manuelle Einfluss des Menschen im wesentlichen auf den Ein- und Ausschaltvorgang. Jedoch legt der Mensch das Ziel des automatischen Vorgangs, also seine musikalische Intention, durch die vorab notwendige Programmierarbeit präzise fest." [5]

Danach wäre es die intentionale Vorformung eines "Klanggestaltungsvorgangs", welche den Automaten als Instrument rettet und ihn vom bloßen Wiedergabegerät, dem Phonographen, abgrenzt:

"Ein Gegenstand, ein Werkzeug, ja auch ein geistiges Produkt, zum Beispiel ein Computerprogramm, ist genau dann ein Musikinstrument, wenn die Möglichkeit der körperlichen oder automatischen Steuerung eines musikalischen Klanggestaltungsvorgangs besteht, der Mensch das Instrument also zur realen, klanglichen Produktion von Musik einsetzen kann." [...] "Auch der Musikautomat ist nach dieser Definition ein Musikinstrument, da er eine programmierte musikalische Idee wiedergibt, nicht jedoch das Reproduktionsgerät (wie etwa ein Schallplattenspieler), sofern es ausschließlich der originalgetreuen Wiedergabe einer gespeicherten musikalischen Produktion dient." [6]

Bei dieser Unterscheidung verbleibt ein gewisses Unbehagen: Der Abruf einer gespeicherten musikalischen Struktur in Programmform - so wird postuliert - sei etwas grundsätzlich anderes als der Abruf einer phonographisch gespeicherten Struktur. Dahinter steht vermutlich die Annahme, beim Phonographen bestehe lediglich eine neue Verklanglichung der originalen Aufführung, während der Musikautomat eine jeweils neue 'instrumentale' Aufführung einer abstrakten, operativen Zeichenstruktur vornehme. Die Künstlerrollen des Welte-Mignon Pianolas widerlegen jedoch die Annahme einer grundsätzlichen Differenz: Sie sollten Anfang des 20. Jh. mit einer authentischen Reproduktion des Spiels einer bekannten Künstlerpersönlichkeit die Konkurrenzfähigkeit des Welte-Pianolas gegen das klanglich unterlegene Grammophon stärken. [7] Umgekehrt ist der Aufführungscharakter phonographischer Musik in den bürgerlichen Salons nicht zu übersehen und wird in literarischen Dokumenten (etwa in Thomas Manns "Zauberberg") dokumentiert. 
Damals wurden indessen beide, Musikautomat und Phonograph bis in die 1930er Jahre, wie Volker Straebel mit Blick auf Carlos Chavez feststellt, als Instrumente verstanden:

"In seiner umfassenden Bestandsaufnahme des Einflusses zeitgenössischer technischer Entwicklungen auf die Musik 'Toward A New Music. Music and Electricity' beschrieb Carlos Chavez 1937 Funktion und Wirkung des damals bereits fünfzig Jahre alten Phonographen zusammen mit Selbstspielklavieren unter der Überschrift 'Elektrische Instrumente zur musikalischen Reproduktion'. Zu selbstverständlich war noch die Vorstellung, daß ein Musik hervorbringendes Gerät eben ein Instrument sei." [8]

Gegenüber dem Phonographen standen die Musikautomaten allerdings den vertrauten Instrumenten allein dadurch näher, dass ihre Mechanismen der Klangerzeugung unmittelbar auf diesen aufsetzten. In der Regel wurden einfach technische Steuerkonfigurationen in gängige Spielinstrumente eingebracht, teilweise konnten die Steuerungen - wie beim PianoVorsetzer - auch wieder entfernt und das Instrument 'normal' gespielt werden. Die Vorstellung spielbarer Musikautomaten - also hybrider Automateninstrumente - lag daher nicht fern und ist in der vielfach implementierten agogischen und dynamischen Steuerbarkeit der PlayerPianos bzw. Vorsetzer realisiert worden. (s. Bild 1) Folgerichtig wurde die Leistungsfähigkeit dieser neuen Instrumente auch in öffentlichen Konzerten erprobt.(Bild 2) Die Vorstellung eines Klavierkonzerts, dessen Solist ein Automat ist, der wiederum von einem künstlerischen Operator kontrolliert wird, scheint uns heute weit entfernt zu sein. Drummachines, Arpeggiatoren, Begleitautomaten oder live-Sequenzing schreiben jedoch genau diese Praxis fort. Die Aufführungskultur hybrider Instrumente der Jahrhundertwende ins 20. Jahrhundert kann daher als Vorgeschichte aktueller Praxis verstanden werden.

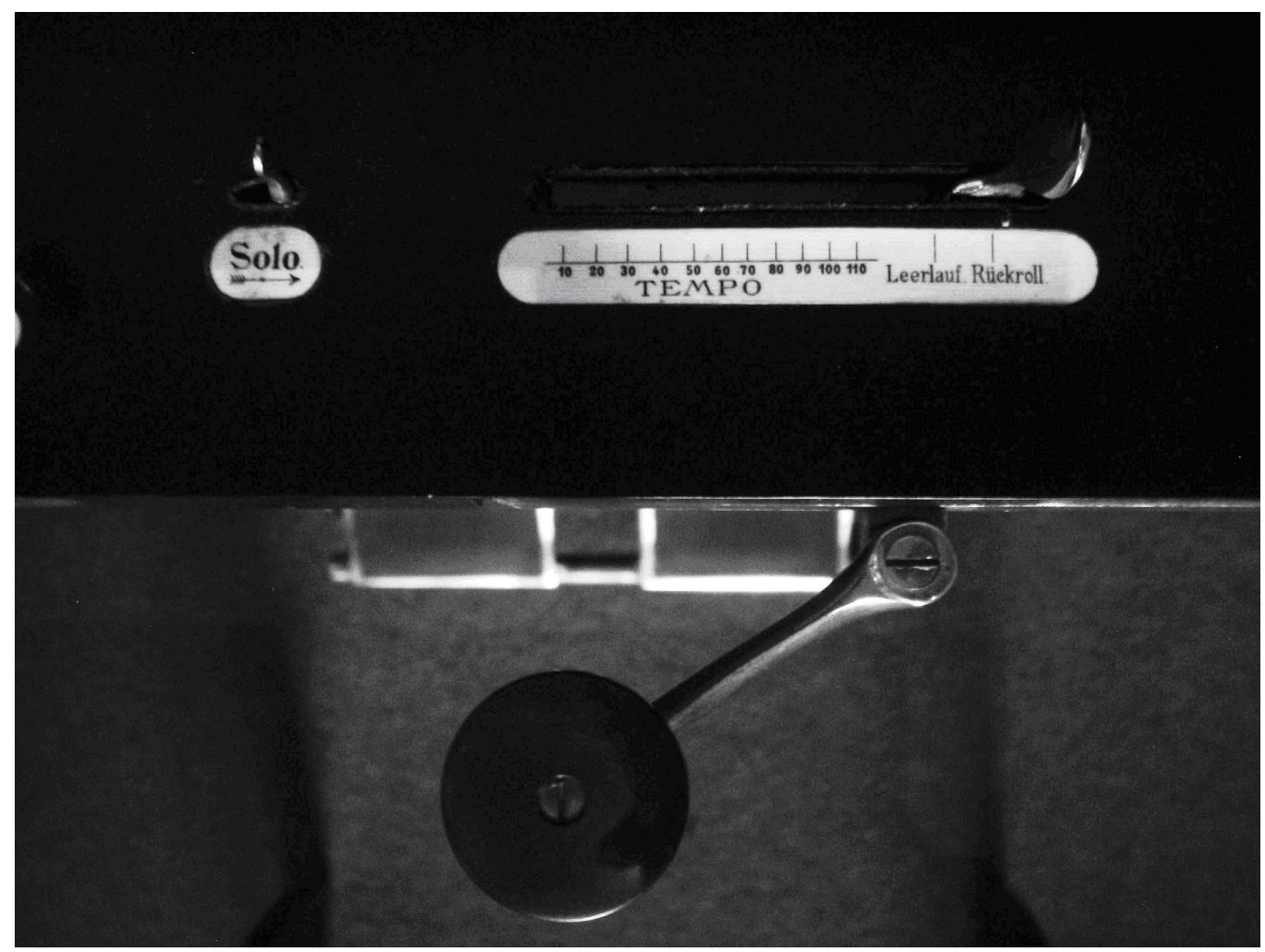

Bild 1: Steuermechanismus eines Piano-Vorsetzers (Detail) 


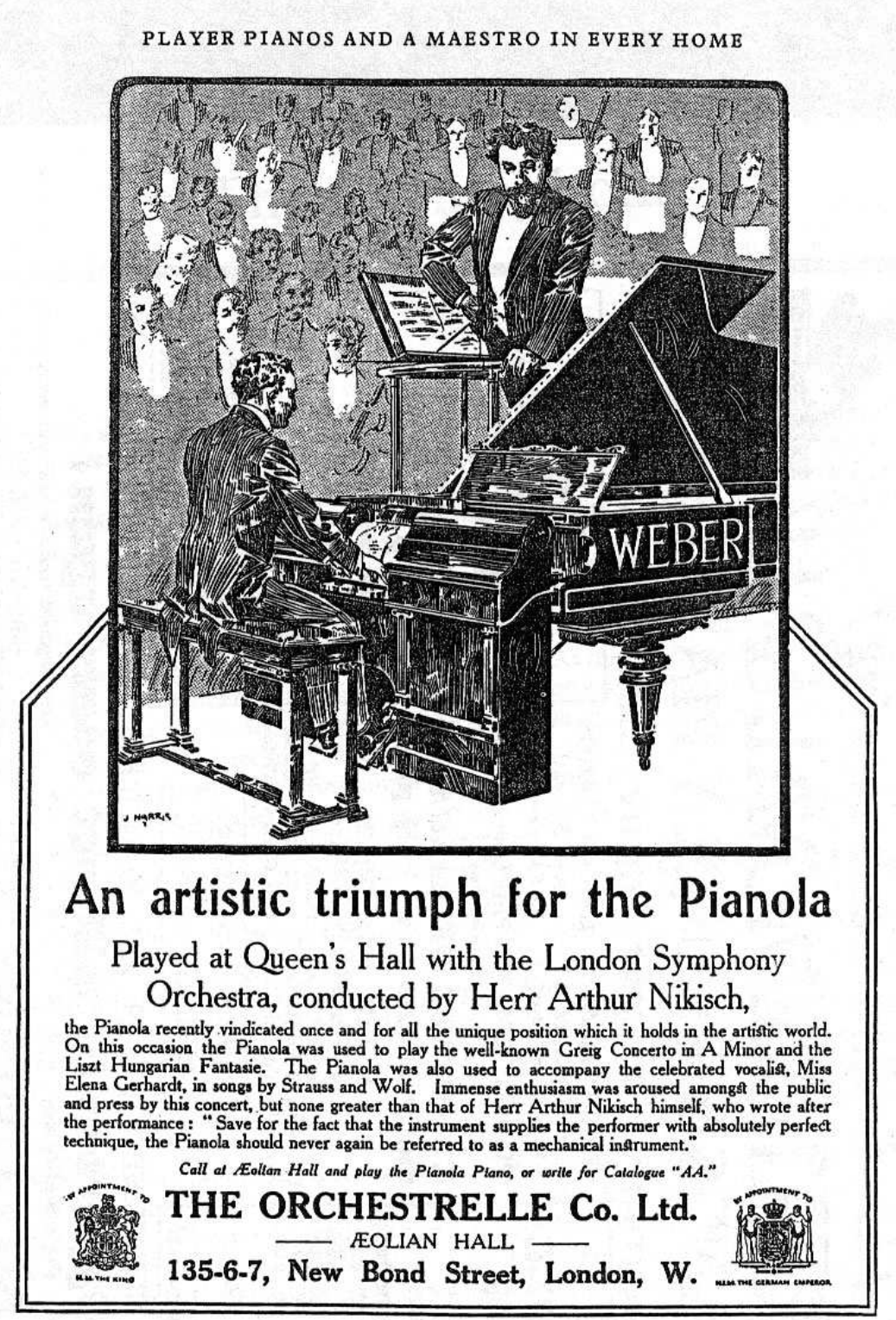

Bild 2: "the instrument supplies the performer with absolutely perfect technique"

Wie die automatische Steuerung von Instrumenten hat auch die phonographische Reproduktion ihre spielerische Vorgeschichte. $\mathrm{Zu}$ den ersten Versuchen der Spielbarkeit der phonographischen Technik gehört László Moholy-Nagys Idee des Phonographen als "Generalin-strument", "das alle bisherigen Instrumente überflüssig macht." [9] Interessanterweise waren seine Visionen von dem Gedanken einer universell einsetzbaren Erzeugung von Klängen geleitet, also eher der Klangsynthese verwandt als den Vorgängen der Speicherung und Ab-bildung. Wenig später folgten die Experimente Ernst Tochs und Paul Hindemiths, die 1930 während des Festivals "Neue Musik Berlin" (der nach Berlin verlegten zunächst Donau-eschinger, dann Baden-Badener Kammermusiktage) vorgestellt wurden. [10] Sie betrafen die kompositorisch motivierte Zeit- und Tonhöhenmanipulation aufgezeichneter Musik und nahmen bereits zentrale gestalterische Verfahren der späteren Tape Music voraus. Dennoch ließ der nächste große Schritt der Reproduktionsmusik, der schließlich das ausgehende 20. Jahrhundert in einer breiten musikalischen Praxis von Unterhaltungsmusik bis zur Avantgarde prägen sollte, noch auf sich warten. Diese neue Praxis, der Zugriff der DJCulture auf kulturelle Archive, also eine Echtzeitmontage sedimentierter Medieninhalte, 
brauchte schon deshalb einige weitere Jahrzehnte bis zu ihrer Durchsetzung als ästhetisches Verfahren, weil sich zunächst die sekundären Medienarchive der persönlichen und subkulturellen Medien-nutzung bilden mussten. Ebenfalls im Wege waren die ästhetischen Normen der europäischen Kunstmusik wie Autorschaft, Werk und Originalität, die in einer zunehmend globalisier-ten Medienkultur relativiert und verändert wurden.

\section{HiFi - ein historisches Zwischenspiel der medialen Abbildung}

Vor der Wiedergeburt der spielbaren Musikautomaten in der Musikelektronik und der spielbaren Phonographen in der DJ-Culture steht als historisches Zwischenspiel eine Phase des Dogmas der authentischen Abbildung: Der Musikautomat der Künstlerrollen verliert seine Daseinsberechtigung angesichts der technischen Perfektionierung der Phonographie, die nun selbst als bestaunenswerte Konfiguration der High Fidelity die Illusion perfekter Abbildung befördert.

"In den dreißiger Jahren vollzog sich also eine Umdeutung der Schallreproduktion vom Instrument zum Medium. Der Hörer erlebt nicht mehr das Entstehen des Klanges in räumlicher und zeitlicher Gegenwart, wie dies in gewisser Weise bei mechanischen Musikinstrumenten noch der Fall ist, sondern versteht das Abspie-len der Schallplatte, dem Betrachten eines Photos vergleichbar, als Verweis auf ein vergangenes Geschehen. So wurde die unverborgene Künstlichkeit des Wiedergabegerätes, dessen technische Beschränkung zu besonderen, 'mediengerechten' Arrangements und Kompositionen geführt hatte, ersetzt durch die Illusion, einem Konzert beizuwohnen. Mit diesem 'Transportieren von Klängen' entwirft das Medium seine eigene Realität. Es entsteht eine Musik ohne Ort." [11]

Diese Ortlosigkeit läßt sich am Bild des mit geschlossenen Augen lauschenden HiFi-Hörers, der sich statt im Wohnzimmer im Konzertsaal wähnt, leicht nachvollziehen. [12] Die Rezeption von phonographisch gespeicherter Musik gerät mit immer neuen technischen Innovationen der Übertragung, Speicherung und Wiedergabe (z.B. FM-Hörfunk, elektromagnetische Tonabnehmer, Stereo, Vinyl-LP etc.) in eine Phase des Ideals der Übereinstimmung von Realem und medialer Abbildung. Diese Situation verändert sich jedoch - teils wiederum getrieben durch studiotechnische Innovationen - schon bald durch Produktionen der populären Musik, welche auf offensichtliche Studioeffekte und manipulationen ohne reale Vorbilder setzen. Im letzten Drittel des 20. Jahrhunderts führen neue soundgestalterische popmusikalische Stile (z. B. Psychedelic) und die phonographische Revolution des Rap zur Repositionierung des HiFi-Ideals als einer möglichen Rezeptionshaltung unter vielen anderen. Die ganz selbstverständlich mit phonographischer Medienproduktion aufgewachsenen Generationen erwarten immer weniger eine 'naturgetreue' Abbildung einer vergangenen Aufführung als vielmehr ein Medienprodukt, das auf die Anforderungen seiner eigenen Aufführungssituation hin optimal produziert ist. Von der Kopplung an eine 'reale Original-Aufführung' verselbständigt sich die Medien-Aufführung mit ihren eigenen spezifischen konventionalisierten Erwar-tungen und Kommunikaten. Die zunächst empfundene Fremdheit und Künstlichkeit der phonographischen Räume schlägt um in die Erwartung ihrer Gestaltbarkeit jenseits 'natürlicher' Referenzen. Mit der zunehmenden Konventionalisierung der sich im Anschluss bildenden neuen Dispositive der Rezeption (Club und Event; Beipiel love parade) und medialen Gestaltung emanzipiert sich eine eigene ästhetische Realität des Mediums, die vormalige Reproduktion erhält selbst Aufführungscharakter. Anstelle des Hier und Jetzt der ursprünglichen liveAufführung ist das Hier und Jetzt der Medienaufführung getreten, die Aura des musikalischen Ritus verschwindet nicht, wie Walter Benjamin vermutete, sondern wird in einer technikkulturellen Konfiguration der Aufführung neu inszeniert. $\mathrm{Zu}$ den auratischen 
Gegenständen dieser neu-en Aufführungskultur gehören - wie in der traditionellen Praxis auch - entsprechend die neuen Instrumente der Performance, statt Violine, Klarinette oder EGitarre erscheinen Plat-tenspieler, Synthesizer und Sampler als Medieninstrumente.

\section{Reproduktionsmusik II - konkrete Experimente und DJ-Sets}

Den Akteuren einer Musik der Reproduktion waren die tief greifenden Veränderungen nicht entgangen, die sich durch die Medien für die musikalische Kultur und damit auch für das Konzept des Musikinstruments vollzogen. Die damit mögliche neue Musik und Musikpraxis wollte erprobt und diskutiert werden, in Europa etwa durch die musique concrète und die Schriften Pierre Schaeffers, der präzise feststellt:

"Alle diese Bemühungen um eine Erneuerung der Musik, in Amerika wie in Europa, bei den Seriellen wie bei den Konkreten, zeichneten sich trotz ihrer Unterschiede durch eine Anzahl von gemeinsamen Kennzeichen aus, deren Bilanz wir wie folgt zogen:

- Der Begriff 'Musikinstrument' ist in Frage gestellt;

- die hergebrachte Notation erweist sich sehr oft als ungenügend zur Darstellung von angestrebten und verwirklichten Klangeffekten;

- die traditionellen Beziehungen zwischen Komponist, Ausführendem und Publikum sind weitgehend außer Kraft gesetzt." [13]

Mit den von ihm selbst und Jacques Poullin Anfang der 1950er entworfenen Magnetbandinstrumenten Phonogène (das eine chromatische Transposition aufgenommener Klänge er-laubt) und Morphophone (ein Bandechogerät) erprobte Schaeffer die neuen Möglichkeiten. [14] Der breite Durchbruch für die Instrumente der Reproduktion in den Alltag musikalischer Gestaltung fand jedoch weder in der seriellen Musik noch in der musique concrète statt, sondern geht von der simulativen Praxis der Heimorgeln (Vorgeschichte der Sampler: Chamberlin und Mellotron) und - ästhetisch weitaus innovativer - von den Turntables der jamaikanischen Soundsystems aus. Die prophezeite Neudefinition des Musikinstruments wird schließlich im hybriden transkulturellen Feld des Rap, Hiphop und Techno greifbar, in dem die traditionelle Notenschrift keine wesentliche Rolle mehr spielt und Kategorien wie Werk und Autorschaft ebenso neu gedacht werden. [15] Die Akteure des New Yorker Disco, des Remix und der jamaikanische Soundsystems bedienen sich ohne Rücksicht auf die traditionellen Werte westeuropäischer Kunstmusik der Ar-chive und Maschinen der Reproduktionsmedien. Mit der Etablierung dieser neuen Praxis verschiebt sich auch die Diskussion um das musikalische Koordinatensystem der Akteure und ihrer technischen Konfigurationen: Sind DJs Musiker, sind ihre Turntable-Setups Musikinstrumente? In der DJ-Szene selbst gibt es zu diesem Thema engere und weitere Definitionen. Der Turntablist - als Virtuose des Scratch\&Mix - gilt gegenüber dem "tradititional DJ" unbestritten als Musiker, sein Set wird genauso handwerklich kompetent und künstlerisch sensibel konfiguriert wie die Stimmung und Intonation eines Konzertflügels.

"The new name distinguishes the turntablist from the traditional DJ, someone who plays records but is not typically thought of as a musician. Although turntablists consider themselves - and are - musicians, their originality is sometimes questioned because they perform on machines designed for automatic play-back." [16]

\section{"Wirkliche Instrumente" in der Welt der Computer, Sampler und Programme}


Ende der 1980er Jahre, also noch weit vor der Ära der Laptop-Performance, sieht Serge Dutrieux seinen Macintosh-Rechner als einen Gegenstand, den er auf der Bühne "wie ein wirkliches Instrument" einsetzen möchte. [17] Sein Wunsch richtet sich nicht auf eine bestimmte musikinstrumentale Beschaffenheit, sondern auf die Vorstellung, den DesktopRechner wie ein Musikinstrument zu spielen. Dieses "wirkliche Instrument" repräsentiert im Kern ein kultu-relles Konzept, der Computer soll aus der Welt der Rechner, der technischen Apparate in die Musikwelt der Instrumente wechseln. Ein Musikinstrument - soviel konnten wir bisher als Minimalkonsens feststellen - ist ein spe-zifisches Artefakt, das mit der Intention des Musikhervorbringens verbunden ist. Dieser Minimalforderung würden sowohl der Phonograph als auch der Musikautomat genügen. Musik-instrumente im kulturellen Sinne sind allerdings mehr als nur Musikerzeuger. Entscheidend für ihre Entwicklung ist die Einbindung in die kulturelle Praxis des instrumentalen Spiels von Musik. Eine solche kulturelle Verankerung ist an einer konventionalisierten kommunikativen Rahmung des Spiels eines Instruments zu erkennen, die gleichzeitig die Voraussetzung für eine kollektive Entwicklung von Spieltechniken und musikalischen Gestaltungsoptionen bil-det. Verbreitete Praxis, instrumentenspezifische Schulenbildung, Notation, Virtuosität sind Merkmale des kulturellen Settings eines Musikinstruments. Ein solcher erweiterter Instru-mentenbegriff gehört jedoch noch zu den Desideraten musik- bzw. kulturwissenschaftlicher Forschung. Es könnte daher sinnvoll sein, zunächst nach den Besonderheiten der neuen Apparate und ihrer Nutzung, den Differenzen zum herkömmlichen Spiel zu fragen. Auch hier geht es wiederum um kulturelle und im engeren Sinne ästhetische Differenzierungen, die nur dann getroffen werden können, wenn die ontologische Perspektive, ein Gegenstand sei per se ein Instrument oder nicht, aufgegeben wird. Ein Plattenspieler, sagen wir ein Technics SL 1200, kann im Wohnzimmer eines HiFi-Fans ein Reproduktionsmedium, in den Händen eines Turntablists wie Kid Koala oder eines experimentellen Virtuosen wie Christian Marclay ein konventionelles Musikinstrument, im DJ-Set eines Grandmaster Flash ein Reproduktionsinstrument sein. Ein Preset-Synthesizer in der Hand eines konventionell spielenden Jazzmusi-kers ist trotz höchst komplexer Synthesemechanismen entsprechend ein gewöhnliches Musikinstrument, während er im Setup einer interaktiven Installation eine vollständig andere Funktion wahrnimmt.

Gleichzeitig kommen die bereits angesprochenen Prozesse der Distanzierung buchstäblich ins Spiel: Schon einfache Instrumente verlagern die Klangerzeugung aus dem menschlichen Körper heraus, sie sind erste Schritte zu einer physischen Distanz von Körperklang und Instrumentenklang. Bernd Enders sieht - im oben zitierten Text - die Instrumente entsprechend als organische Erweiterungen des Körpers. Sie sind Teil eines technikkulturellen Prozesses, der die Entwicklung musikalischer Praxis, ihre Formen und Stile von Beginn an begleitet.

Die distanzierten Verhältnisse, von denen der Titel des Beitrags spricht, stellen sich also nicht erst mit den elektronischen Medien oder der Informationstechnologisierung der Musik ein. Sie erreichen jedoch eine neue Qualität, wenn die technikkulturellen Spezifika der Reproduktionsmedien oder der Computermedien als Teil des ästhetischen Prozesses integriert werden. Die präformierte Gestalt und die Form des Zugriffs auf die bereits erwähnte "halbfertige Musik" definieren diese Instrumente mit.

Bei den Reproduktionsmedien ist der gespeicherte Klang auf zweifache Weise Teil des instrumentalen Gestaltungsvorgangs: zum einen als Klangschrift selbst, die unvermittelt analog (z.B. beim Plattenspieler) oder vermittelt digital (z.B. beim Sampler) ausgelesen und so gespielt werden kann; zum anderen als Teil des kulturellen Archivs der phonographischen Produktionen, deren sedimentierte Verweisketten spielerisch neu geknüpft werden können. 
Der Sampler vereinigt als Musikinstrument der digitalen Phonographie beide Aspekte, während sich seine kulturelle ästhetische Tradition aus drei Quellen speist:

- der Simulation traditioneller Instrumente; hierzu gehört der Traum der unbegrenzten in-strumentalen Verfügbarkeit aller Klänge einschließlich der menschlichen Stimme

- der experimentellen Eroberung der Neuen Klanglichkeit der Phonographie; hierzu gehören die Stile der musique concrete, aber auch die Klangexperimente der populären Musik (sowie der Unbefugten und Grenzgänger zwischen den Künsten, Beispiel Christian Marclay)

- der DJ-Culture, deren Gegenstand die ästhetische Aneignung der phonographischen Archive zweiter Ordnung (der Archive der persönlichen und gruppenspezifischen Alltagswelten) ist.

Verständlicherweise wird sich das instrumentale Spiel des Samplers in der Tradition der Simulation auch in der Spielweise an die simulierten Instrumente anlehnen. Das typische Inter-face hierfür wäre das Keyboard, welches als Klaviatur bei Cembalo und Piano oder als OrgelSpieltisch bereits eine vertraute Lösung zur Kontrolle mechanisierter Instrumente darstellt. Als weitere Annäherung an traditionelle Instrumente ist deren technische Erweiterung oder vollständige Ausprägung als Controller, etwa als Blaswandler oder MIDIVioline möglich. Damit sind in in einer früheren Praxis erlernte Spieltechniken auf die medientechnischen Umgebungen übertragbar. Solche Konzepte werden entsprechend als "augmented instruments" oder "extended instruments" bezeichnet. [18] Denkbar ist allerdings auch ein idealer Controller, welcher als universelles Musikinstrument dienen könnte und die Universalität des Sampling auf der Ebene des instrumentalen Interfaces repräsentiert. Tatsächlich findet sich vielfach in der Literatur die Vorstellung eines universellen Controllers als Vision eines zukünftigen Instruments.

Bei algorithmischen Prozessen der programmierten Erzeugung von Klangstrukturen ist der instrumentale Gestaltungsvorgang ebenso auf die Anordnung der Programmteile und ihre Beeinflussung gerichtet. Auch hier gibt es den Simulationsaspekt, etwa bei der BegleitAutomatik von Alleinunterhalterkeyboards oder den scheinbar natürlich verlaufenden Hüllkurven von Klangflächen. Wird dieses Paradigma der Simulation traditioneller Instrumente verlassen, beginnt der vertraute Grund der "Körperinstrumente" brüchig zu werden. Die spielerische Beherrschung oder experimentelle Erprobung der automatischen Prozesse verlangt eine veränderte Kultur des instrumentalen Spiels. Nicht mehr die physische Anordnung der Tasten des Keyboard sind spielerisch zu bewältigen, die generative Struktur der Programme rückt ins Zentrum: "Man muss das Patch lernen wie ein Instrument." [19] Das Programm und seine technische Konfiguration werden Teil des kulturellen Konzepts 'Instrument'.

\section{Mythos Kontrolle}

Eine kritische Perspektive soll abschließend auf eine im Diskurs technischer Musikinstrumente immer wieder mißverstandene Vision gerichtet werden: die Vorstellung einer möglichst vollständigen Kontrolle des Instruments durch den menschlichen 'Operator'. Die empfundene Distanz oder Nähe zu technischen Musikinstrumenten ist nicht eine Frage der mehr oder weniger vollständigen Kontrollierbarkeit von Parametern, sondern der Relation von in-strumentaler Organisation komplexer Strukturen und ästhetischer Strategie des Spiels. Wäh-rend traditionelle Instrumente ihre physische Klangerzeugung als dominante Bestimmungsfaktoren ins Instrumentalspiel einbringen (deshalb sind diese Faktoren zu Recht auch in der Klassifikation der Instrumente so prominent, s.o.) sind elektronische und mehr 
noch digitale Musikinstrumente selbst vorgängig Produkte einer rationalen und rationalisierten Kultur. Sie enthalten als technisch-kulturelle Artefakte die Ratio musikalischer Produktion bereits als sedimentierte Struktur. Der Versuch, diese Artefakte einer industriellen und postindustriellen Kontrolle zu Werkzeugen ästhetischer Gestaltung zu machen, indem möglichst viele Parameter präzise kontrollierbar implementiert werden, könnte allerdings Probleme mit sich bringen.

So beruht die instrumentale Anwendbarkeit des Synthesizers in Form des Mini-Moog gerade auf einer Reduktion der Kontrollmöglichkeiten auf einige wenige zentrale Variablen, welche die technologische Komplexität der modularen Synthesizer-Ära eingrenzen und intuitiv handhabbar machen. Erkauft wird die Handhabbarkeit mit dem weitgehenden Verzicht auf die Flexibilität und Parameterkontrolle der modularen Systeme. Dennoch hält sich der My-thos der totalen Kontrolle hartnäckig in Visionen idealer musikalischer Interfaces.

"Je besser kontrollierbar eine Technik ist, desto besser lässt sich diese Technik instrumentalisieren. Je automatischer und unkontrollierbarer die Technik bezie-hungsweise je eingeschränkter das Interface des technischen Apparates beziehungsweise des medialen Dispositivs sowohl auf der Seite der Hardware als auch der Software ist, desto mehr wird sie als Medium bezeichnet." [20]

"Sobald der Klang nicht nur rudimentär, das heißt vielleicht durch einen oder zwei Parametern (sic!), sondern durch mehrere bis fast unendlich viele Parameter kontrollierbar wird, kann von einem 'Musik-Instrument' im herkömmlichen Sinne gesprochen werden." [21]

Genau dies scheint eine von technischer Vernunft beherrschte Instrumenten-Vorstellung zu sein, die weit verbreitet ist und oft noch zusammen mit der Forderung nach optimaler Ergonomie auftritt. Damit lässt sich das Paradigma herkömmlicher Instrumente kaum in Richtung der neuen Medientechnologien überschreiten, weniger noch, da auch die herkömmlichen Instrumente sich weder durch eine perfekte Kontrolle aller Parameter noch durch beste Ergonomie auszeichnen. Der britsche Elektronica-Produzent, DJ und Bassist Squarepusher (Tom Jenkinson) sieht hier ein dialektisches Verhältnis zwischen der Eigendynamik technischer Konfigurationen, die er als kulturelle Hervorbringungen versteht, und der menschlichen Intentionalität künstlerischer Schöpfung. Seine Position beschreibt eine "Dialektik der Aufklä-rung" elektronischer und digitaler Musikinstrumente ("the machine"): Der Mensch scheitert an den von ihm selbst produzierten zweckrationalen Konfigurationen, wenn er sie im Sinne der totalen Beherrschung einsetzen will.

"Trying to force a machine to manifest a conscious purpose brings about a stifling and deadening process that only in our time could pass for 'creativity'. It imposes that the didactic 'collaboration' with a machine is a strictly one-way energy chan-nel, from the user to the machine. In this situation, the machine cannot constitute a genuine 'oppositional factor' in a dialectical equation as it offers not the antithesis of the conscious human will but rather the negation of it. When being forced to 'purpose', all the machine seems to be capable of is resistance. It is not that the machine is a lifeless vacuum that continually absorbs inspiration and ideas from its user, but that the user hinders the collaboration by assuming he is the progenitor of these things in the first place. It is in this trick of perspective, from the hum-ble 'it happened' to the questionable 'I made it happen' to the disastrous 'I can make it happen' that lies the labyrinth of paradoxes that is our 'modern' world. [...] Unfortunately, working with any material in a violent and dictatorial way simply produces artefacts of human stupidity, not art." [22] 
Ein positives Gegenbeispiel aus der elektronischen live-Music wäre die von Michel Waisvisz entwickelte Crackle Box, die für das Instrumenten-Konzept des STEIM (Studio for Electro Instrumental Music) in Amsterdam paradigmatisch ist. Andreas Otto, der sich in seiner Magisterarbeit mit der Geschichte der Entwicklung elektronischer Musikinstrumente am STEIM ausführlich auseinander gesetzt hat, schreibt dazu:

"Kleinste Druckausübungen und Bewegungen der Finger auf der Strom leitenden Oberfläche finden ihre unmittelbare klangliche Umsetzung ohne Zeitverlust oder mechanische Verzögerung. Durch die Berührung der Steuerung kann der Körper des Instrumentalisten als physikalisch widerständiger Teil im elektronischen Schaltkreis des Instruments betrachtet werden. Das Spiel ist intuitiv zu erlernen, das heißt durchzuhören bei der Improvisation und nicht durch logische, visuell geleitete Annäherung, wie es bei der Arbeit mit den Oberflächen klanggenerierender Apparate häufig der Fall ist." [23]

Das Instrument spielt hier gerade nicht die Rolle einer konkreten Ausprägung der Vision totaler Kontrolle, wie sie oft und gerade in digitalen Umgebungen anzutreffen ist, sondern begegnet dem Akteur als gefundenes technologisches Objekt, das durch kleine Modifikationen für einen musikalischen Prozess brauchbar wird, $\mathrm{zu}$ dem es seine Eigenschaften beisteuert. Nun ist die Crackle Box gerade kein Instrument, das eine algorithmische oder phonographische 'halbfertige Musik' steuert. Sie ähnelt in der Einbeziehung des menschlichen Körpers in den Schwingkreis des elektronischen Oszillators eher dem Theremin als aktuellen Synthesizern. Dennoch lehrt sie auch für die neue Welt digitaler musikalischer Interfaces, dass Einfachheit, Unmittelbarkeit und spielerische Überraschung mehr Nähe und Ideenreichtum produzieren können als die distanzierten Verhältnisse rationaler Kontrolle. 


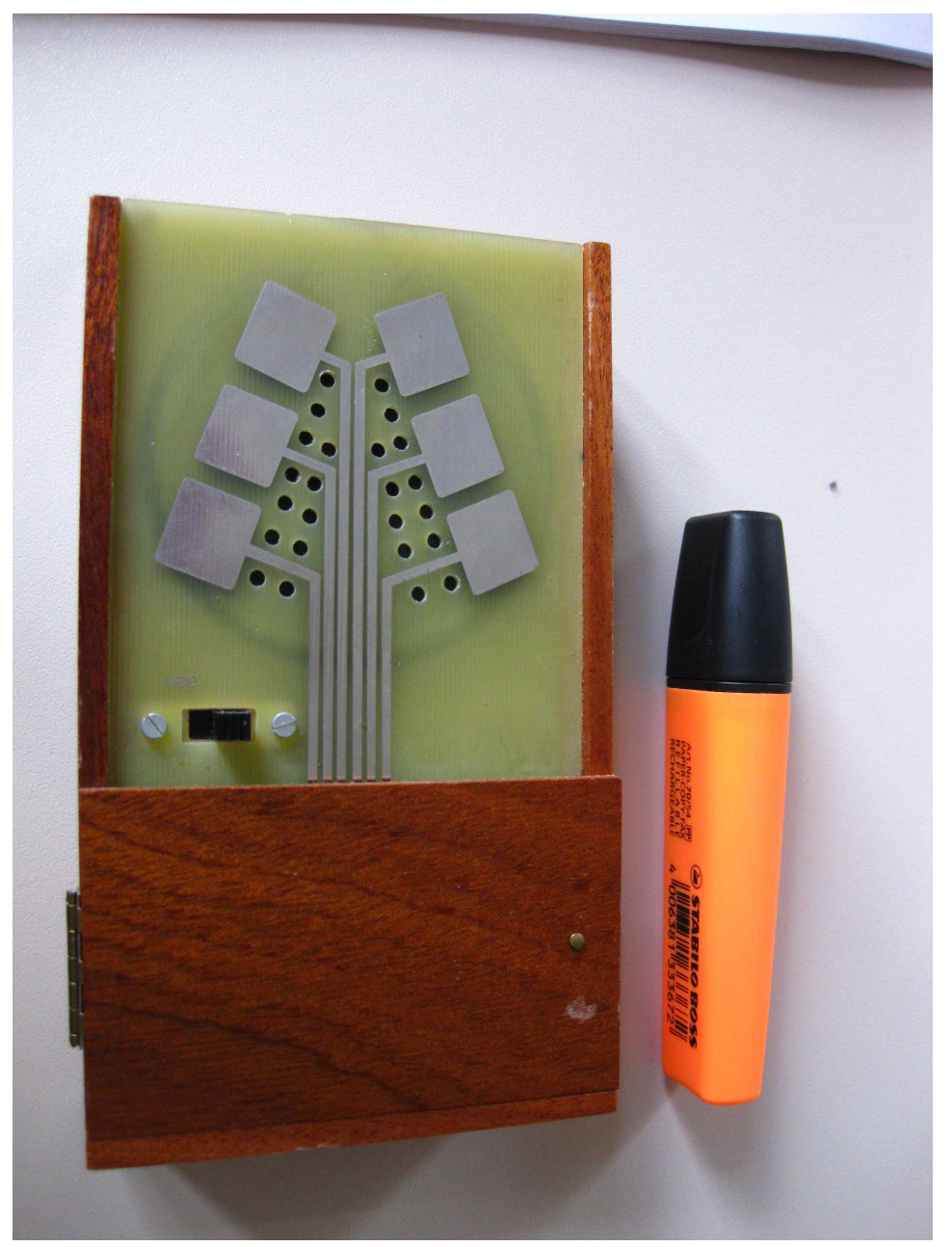

Bild 3: CrackleBox, Originalgröße 14x8cm

Es bleiben, zusammenfassend gesagt, zwei wichtige Desiderate: Es wäre eine kulturelle Definition des Musikinstruments unter Einbezug der technisch-medialen Konfigurationen weiter zu entwickeln. In einem weiteren Schritt wären die Instrumente und die neue ästhetische Praxis (die weit über die Tradition der westeuropäischen Kunstmusik hinausgeht und die Formen populärer Musik in den Medien einschließt) in Beziehung zu setzen. Insgesamt könnte so ein besseres Verständnis des (medien-)musikalischen Wandels gewonnen werden.

Auch die neuen Instrumente einer technischen Kultur dürften sich in einer ähnlichen Weise etablieren wie die klassischen Instrumente: Einer Phase der Fremdheit und Exploration folgt eine Phase der Komplexreduktion und die Ausbildung einer musikalischen Sprache des jeweiligen Instruments, falls sich die jeweilige Konfiguration in einer breiteren Praxis etablieren kann. Schließlich entsteht - wie an der Verwendung traditioneller Instrumente in der zeitge-nössischen Musik des 20. Jahrhunderts zu beobachten ist - wiederum eine Phase der Dis-tanz, der neuerlichen Rationalisierung und eine darauf aufbauende neue Praxis. Eine breite Phase der Exploration liegt bereits hinter uns, mehr noch, mit DJ -Set und Sampler verbinden sich längst etablierte Formen und Stile. Wie die Dynamik der Simulation und Virtualisierung in den digitalen Medien verraten, sind jedoch weder die technischen Konfigurationen noch ihre kulturelle Aneignung ausentwickelt. 
Der Diskurs, was ein musikalisches Instrument ausmacht, bleibt in dieser Situation weiter offen. Die direkte Beeinflussung von Parametern in der Zeit scheint mir eine Kategorie zu sein, die übergreifend konsensfähig ist. Ebenso erscheint es notwendig, Aspekte des intuitiven Lernens bzw. Übens und der musikkulturellen Etablierung miteinzubeziehen. Demge-genüber sind Kategorien der umfassenden Kontrolle zurückzustellen. Die technikkulturelle Entwicklung der eingeführten Instrumente zeigt, dass dagegen die Optionen einer Ausdifferenzierung des Klangs und der weiten musikstrukturellen Anwendbarkeit eine zentrale Rolle spielen. Dies könnte auch für Medieninstrumente relevant sein.

\section{Fußnoten}

1. von Hornbostel, Erich M. / Sachs, Curt: Systematik der Musikinstrumente. Ein Versuch. In: Zeitschrift für Ethnologie 46, 1914 (4-5), S. 553

2. Tellef Kvifte hat in seinem Versuch einer Erweiterung der klassischen Instrumentensystematik auf die "Instrumente des elektronischen Zeitalters" entsprechend den Aspekt der Spieltechnik in den Vordergrund gerückt. S. Kvifte, Tellef: Instruments and the Electronic Age. Toward a Terminology for a Unified Description of Playing Technique. Oslo 1989.

3. Enders, Bernd: Instrumentenkunde - Form, Funktion und Definition des Musikinstruments im Spannungsfeld von Musik und Technik, in: Edler, Arnfried; Helms, Siegmund und Hopf, Helmuth (Hg.): Musikpädagogik und Musikwissenschaft. Florian Noetzel Verlag, Wilhelmshaven 1987, S. 306345, hier S. 318.

4. Miyazaki, Shintaro: Medien, ihre Klänge und Geräusche - Medienmusik vs. / (=) Instrumentalmusik. In: PopScriptum 9 - Instrumentalisierungen - Medien und ihre Musik. Schriftenreihe hg. vom Forschungszentrum Populäre Musik der Humboldt-Universität zu Berlin 2008, S. 3 [http://www2.huberlin.de/fpm/popscrip/themen/pst09/Miyazaki.pdf, 1.3.2009].

5. Enders 1987, S. 321.

6. Enders 1987, S. 336f.

7. Bei den Künstlerrollen wurde das Spiel eines Künstlers (z.B. Max Reger) über die Bewegungen der Klaviermechanik aufgezeichnet und auf eine Pianola-Steuerrolle übertragen.

8. Straebel, Volker: Klangraum und Klanginstallation. Klanginstallation zwischen elektroakustischer Technik, Performance und Skulptur. In: de la Motte-Haber, Helga (Hg.), Klangkunst. Katalog zu Sonambiente. Festival für Hören und Sehen der Akademie der Künste Berlin, München 1996, S. 219 221, hier S. 219.

9. Moholy-Nagy, László: MUSICO-MECHANICO, MECHANICO-OPTICO. In: Stuckenschmidt, HansHeinz (Hg.): Musik und Maschine: Sonderheft der Musikblätter des Anbruch, Heft 8/9. S.363-367, hier S. 365.

10. Laut Programm als "Originalwerke für Schallplatten"; s.a. Katz, Mark: Capturing Sound: How Technology Has Changed Music. Berkeley: University of California Press 2004, S. 99ff.

11. Straebel 1996, S. 219.

12. S. dazu auch Blaukopf, Kurt: Musik im Wandel der Gesellschaft. Grundzüge der Musiksoziologie. München 1982. Die Künstlichkeit der phonographischen Räume ist eine der zentralen Eigenschaften der "Mediamorphose" bei Kurt Blaukopf und wird dort ausführlich thematisiert.

13. Schaeffer, Pierre: Musique concrète: Von den Pariser Anfängen um 1948 bis zur elektroakustischen Musik heute. Stuttgart 1974, S. 29. 
14. Zu den Instrumentkonfigurationen der analogen Phase der Reproduktionsmusik s. Klages, Thorsten: Medium und Form - Musik in den (Re-)Produktionsmedien. Osnabrück 2002.

15. Tricia Rose zeigt in ihrem Standardwerk zum Hiphop (Black Noise. Rap Music and Black Culture in Contemporary America. Wesleyan University Press 1994), dass Originalität und Autorschaft auch im Hiphop eine wichtige Rolle spielen, jedoch nicht im klassischen westeuropäischen Sinne.

16. Katz 2004, S. 116.

17. Serge Dutrieux, zitiert nach Großmann, Rolf: "Sechs Thesen zu musikalischen Interfaces". In: Klaus Peter Dencker (Hg.), Interface 2. Hamburg 1995, S. 155-162, hier S. 158.

18. S. dazu Eduardo R. Miranda / Marcelo M. Wanderley: New Digital Musical Instruments: Control and Interaction Beyond the Keyboard. Middleton: A-R Editions 2006, S. 20f. sowie Jin Hyun Kim / Uwe Seifert: Embodiment: The Body in Algorithmic Sound Generation. In: Contemporary Music Review, Vol. 25. No. 1/2, 2006, S. 140f.

19. Christian Fennesz im Interview: Fennesz "Organisch elektronisch Fennesz" in Keys 11/2007, S. 106107, hier 106.

20. Miyazaki 2008, S. 4.

21. Miyazaki 2008, S. 5.

22. Tom Jenkinson "Collaborating with Machines" In: Flux Magazine, March 2004, zit. nach http://www.warprecords.com/?news=789, 1.6.2007.

23. Otto, Andreas: Die Entwicklung elektronischer Musikinstrumente am STEIM (Studio für elektroinstrumentale Musik) in Amsterdam seit 1969. Manuskript (Magisterarbeit) Lüneburg 2008, S. 20. 